\title{
Hyers-Ulam-Rassias Stability Criteria of Nonlinear Differential Equations of Lane-Emden Type
}

\author{
Maher Nazmi Qarawani \\ Department of Mathematics, Al-Quds Open University, Salfit, West-Bank, Palestine \\ mkerawani@qou. edu
}

\begin{abstract}
In this paper we establish Hyers-Ulam-Rassias stability and Hyers-Ulam Criteria for second order non-linear ordinary differential equations of Lane-Emden type; moreover two examples of such equations are considered.
\end{abstract}

Keywords: Hyers-Ulam-Rassias Stability, Nonlinear Differential Equations, Lane-Emden Type.

MSC: 34Cxx , 34K20, 37C75.

\section{Introduction}

Equations of the Lane-Emden type arise in mathematical physics and astrophysics. These Equations describe the temperature variations of a spherical gas cloud under the mutual attraction of its molecules and subject to the law of classical thermodynamics, [17].

The objective of this article is to investigate the Hyers-Ulam-Rassias Stability for the Lane-Emden type equation

$$
u^{\prime \prime}(t)+\left(\frac{n-1}{t}\right) u^{\prime}+g(t) f(u)=h(t)
$$

and the nonlinear differential equation of second order

$$
u^{\prime \prime}(t)+\left(\frac{n-1}{t}\right) u^{\prime}+g(t) f(u)=0
$$

with the initial conditions.

$$
u\left(t_{0}\right)=u_{0}, u^{\prime}\left(t_{0}\right)=u_{1}
$$

Moreover in this paper we consider the Hyers-Ulam-Rassias Stability for the Emden-Fowler equation

$$
u^{\prime \prime}(t)+\left(\frac{2}{t}\right) u^{\prime}+g(t) f(u)=0
$$

Here $n$ is a real parameter such that $n>2, t \in\left[t_{0}, T\right], 0 \leq t_{0}<T<\infty$, $h(t):[0, \infty) \rightarrow R, g(t):[0, \infty) \rightarrow R$ are continuous. Suppose that there is $L>0$ such that

$$
|f(u(t))-f(v(t))| \leq L|u-v|
$$

In 1940, Ulam [27] posed the stability problem of functional equations. In the talk, Ulam 
discussed a problem concerning the stability of homomorphisms. A significant breakthrough came in 1941, when Hyers [5] gave a partial solution to Ulam's problem. During the last two decades very important contributions to the stability problems of functional equations were given by many mathematicians see $[2,6-8,13,18-20,23]$. More than twenty years ago, a generalization of Ulam's problem was proposed by replacing functional equations with differential equations: The differential equation $F\left(t, y(t), y^{\prime}(t), \ldots, y^{(n)}(t)\right)=0$ has the Hyers-Ulam stability if for given $\varepsilon>0$ and a function $y$ such that

$$
\left|F\left(t, y(t), y^{\prime}(t), \ldots, y^{(n)}(t)\right)\right| \leq \varepsilon
$$

there exists a solution $y_{0}$ of the differential equation such that

and $\lim _{\varepsilon \rightarrow 0} K(\varepsilon)=0$.

$$
\left|y(t)-y_{0}(t)\right| \leq K(\varepsilon)
$$

The first step in the direction of investigating the Hyers-Ulam stability of differential equations was taken by Obloza see [16, 17]. Thereafter, Alsina and Ger [1] have studied the Hyers-Ulam stability of the linear differential equation $y^{\prime}(t)=y(t)$. The Hyers-Ulam stability problems of linear differential equations of first order and second order with constant coefficients were studied in the papers ([11, 28]) by using the method of integral factors. The results given in [9, 24, 25] have been generalized by Popa and Rus [21, 22] for the linear differential equations of nth order with constant coefficients. Gordji et al. [4] get sufficient conditions for Hyers-Ulam stability of the first order and the second order nonlinear partial differential equations. Lungu and Craciun [12] established results on the Ulam-Hyers stability and the generalized Ulam-Hyers-Rassias stability of nonlinear hyperbolic partial differential equations.

In addition to above-mentioned studies, several authors have studied the Hyers-Ulam stability for differential equations of first and second order see $[3,10,14,26]$.

\section{Preliminaries}

We introduce some definitions as follows:

Definition 2.1 We say that equation (1.1) ( or (1.2) with $h(t) \equiv 0$ ) has the Hyers-Ulam-Rassias (HUR) stability with respect to $\varphi$ if there exists a positive constant $k>0$ with the following property: For each $u(t) \in C^{(2)}\left(\left[t_{0}, \infty\right),(-\infty, \infty)\right)$, if

$$
\left|u^{\prime \prime}(t)+\left(\frac{n-1}{t}\right) u^{\prime}+g(t) f(u)-h(t)\right| \leq \varphi(t),
$$

then there exists some $z_{0}(t)$ of the equation (1.4) such that

$$
\left|u(t)-z_{0}(t)\right| \leq k \varphi(t)
$$

Definition 2.2 We say that equation (1.4) has the Hyers-Ulam-Rassias (HUR) stability with respect to $\varphi$ if there exists a positive constant $k>0$ with the following property: For each

$$
\begin{aligned}
& u(t) \in C^{(2)}\left(\left[t_{0}, \infty\right),(-\infty, \infty)\right), \text { if } \\
& \qquad\left|u^{\prime \prime}(t)+\left(\frac{2}{t}\right) u^{\prime}+g(t) f(u)\right| \leq \varphi(t),
\end{aligned}
$$


Hyers-Ulam-Rassias Stability Criteria of Nonlinear Differential Equations of Lane-Emden Typ

then there exists some $z_{0}(t)$ of the equation (1.4) such that

$$
\left|u(t)-z_{0}(t)\right| \leq k \varphi(t)
$$

Lemma 2.1 (Gronwall's Inequality) Let $\mathrm{u}(\mathrm{t})$ and $\mathrm{v}(\mathrm{t})$ be nonnegative continuous functions on some interval $0<t_{0} \leq t \leq t_{0}+a$. Also, let the function $f(t)$ be positive, continuous, and monotonically nondecreasing on $\left[t_{0}, t_{0}+a\right]$ and satisfy the inequality

$$
u(t) \leq f(t)+\int_{t_{0}}^{t} u(s) v(s) d s
$$

then, there holds the inequality

$$
u(t) \leq f(t) \exp \left(\int_{t_{0}}^{t} u(s) d s\right), \text { for } t_{0} \leq t \leq t_{0}+a
$$

\section{Main results on Hyers-Ulam-Rassias Stability}

Theorem 3.1 Suppose that $u(t) \in C^{(2)}\left(\left[t_{0}, T\right],(-\infty, \infty)\right)$ satisfies the inequality (2.1) with initial conditions (1.3). Let $\varphi(t):\left[t_{0}, \infty\right) \rightarrow(0, \infty)$ be a continuous function such that

$$
\int_{t_{0}}^{t} s^{1-n} \int_{t_{0}}^{s} r^{n-1} \varphi(r) d r d s \leq C \varphi(t), \quad \forall t \geq t_{0} .
$$

If (1.5) holds, then the solution of (1.1) is stable in the sense of HUR.

Proof. Let $\varepsilon>0$ and $u(t)$ be an approximate solution of the initial value problem (1.1),(1.3). We will show that there exists a function $z_{0}(t) \in C^{(2)}\left(\left[t_{0}, \infty\right),(-\infty, \infty)\right)$

satisfying (1.1) and (1.3) such that

$$
\left|u(t)-z_{0}(t)\right| \leq k \varphi(t)
$$

The inequality (2.1) implies that

$$
-\varphi(t) \leq u^{\prime \prime}(t)+\left(\frac{n-1}{t}\right) u^{\prime}+g(t) f(u)-h(t) \leq \varphi(t),
$$

Multiplying (3.2) by $t^{n-1}$, we get

$$
-t^{n-1} \varphi(t) \leq\left(t^{n-1} u^{\prime}(t)\right)^{\prime}+t^{n-1} g(t) f(u)-t^{n-1} h(t) \leq t^{n-1} \varphi(t),
$$

Integrating (3.3) from $t_{0}$ to $t$, we have

$$
\begin{aligned}
-\int_{t_{0}}^{t} s^{n-1} \varphi(s) d s & \leq t^{n-1} u^{\prime}(t)-t_{0}{ }^{n-1} u_{1}+\int_{t_{0}}^{t} s^{n-1} g(s) f(u(s)) d s-\int_{t_{0}}^{t} s^{n-1} h(s) d s \\
& \leq \int_{t_{0}}^{t} s^{n-1} \varphi(s) d s,
\end{aligned}
$$

Or, equivalently we get 


$$
\begin{array}{r}
-t^{1-n} \int_{t_{0}}^{t} s^{n-1} \varphi(s) d s \leq u^{\prime}(t)-t^{1-n} t_{0}{ }^{n-1} u_{1}+t^{1-n} \int_{t_{0}}^{t} s^{n-1} g(s) f(u(s)) d s \\
-t^{1-n} \int_{t_{0}}^{t} s^{n-1} h(s) d s \leq t^{1-n} \int_{t_{0}}^{t} s^{n-1} \varphi(s) d s,
\end{array}
$$

Again integrating the last inequality from $t_{0}$ to $t$, we obtain

$$
\begin{aligned}
\mid u(t)-u_{0}-t^{2-n} t_{0}{ }^{n-1} u_{1}+t_{0} u_{1} & +\int_{t_{0}}^{t} s^{1-n} \int_{t_{0}}^{s} r^{n-1} g(r) f(u(r)) d r d s \\
& -\int_{t_{0}}^{t} s^{1-n} \int_{t_{0}}^{s} r^{n-1} h(r) d r d s \mid \leq \int_{t_{0}}^{t} s^{1-n} \int_{t_{0}}^{s} r^{n-1} \varphi(r) d r d s
\end{aligned}
$$

It is clear to see that

$$
\begin{aligned}
z_{0}(t)=u_{0}-t_{0} u_{1}+t^{2-n} t_{0}{ }^{n-1} u_{1} & -\int_{t_{0}}^{t} s^{1-n} \int_{t_{0}}^{s} r^{n-1} g(r) f\left(z_{0}(r)\right) d r d s \\
& -\int_{t_{0}}^{t} s^{1-n} \int_{t_{0}}^{s} r^{n-1} h(r) d r d s
\end{aligned}
$$

satisfies the Eq. (1.1) with the initial condition (1.3).

Now consider the difference

$$
\begin{aligned}
\left|u(t)-z_{0}(t)\right| \leq \mid u(t) & -u_{0}-t^{2-n} t_{0}{ }^{n-1} u_{1}+t_{0} u_{1}+\int_{t_{0}}^{t} s^{1-n} \int_{t_{0}}^{s} r^{n-1} g(r) f(u(r)) d r d s \\
& -\int_{t_{0}}^{t} s^{1-n} \int_{t_{0}}^{s} r^{n-1} h(r) d r d s|+| \int_{t_{0}}^{t} s^{1-n} \int_{t_{0}}^{s} r^{n-1} g(r) f(u(r)) d r d s \\
& -\int_{t_{0}}^{t} s^{1-n} \int_{t_{0}}^{s} r^{n-1} g(r) f\left(z_{0}(r)\right) d r d s \mid \\
\leq C \varphi(t) & +\int_{t_{0}}^{t} s^{1-n} \int_{t_{0}}^{s} r^{n-1}|g(r)|\left|f(u(r))-f\left(z_{0}(r)\right)\right| d r d s \\
\leq & C \varphi(t)+L M \int_{t_{0}}^{t} s^{1-n} \int_{t_{0}}^{s} r^{n-1}\left|u(r)-z_{0}(r)\right| d r d s
\end{aligned}
$$

where $\quad M=\max _{t_{0 \leq t \leq T}}|g(t)|$.

Applying Gronwall's inequality to (3.4) we infer that 
Hyers-Ulam-Rassias Stability Criteria of Nonlinear Differential Equations of Lane-Emden Typ

$$
\begin{aligned}
\left|u(t)-z_{0}(t)\right| & \leq C \varphi(t) \exp \left(L M \int_{t_{0}}^{t} s^{1-n} \int_{t_{0}}^{s} r^{n-1} d r d s\right) \\
& \leq C \varphi(t) \exp \left(\frac{L M}{n} \int_{t_{0}}^{t} s^{1-n}\left(s^{n}-t_{0}{ }^{n}\right) d s\right) \\
& \leq C \varphi(t) \exp \left(\frac{L M}{n} \int_{t_{0}}^{t} s^{1-n} s^{n} d s\right)=C \varphi(t) \exp \left(\frac{L}{n} \int_{t_{0}}^{t} s d s\right) \\
& \leq C \varphi(t) \exp \left(\frac{L M\left(t^{2}-t_{0}{ }^{2}\right)}{2 n}\right) \leq C \varphi(t) \exp \left(\frac{L M T^{2}}{2 n}\right) \equiv k \varphi(t)
\end{aligned}
$$

Consequently we have

$$
\left|u(t)-z_{0}(t)\right| \leq k \varphi(t)
$$

which completes the proof of Theorem 3.1.

Corollary 3.1 Replacing $\varphi(t)$ by $\varepsilon$ in the inequality (3.2) we can get Hyers-Ulam stability for Eq. (1.1) in the interval $0<t_{0} \leq t \leq T$, i.e. if $u(t) \in C^{(2)}\left[t_{0}, T\right]$ satisfies (1.5) and

$$
\left|u^{\prime \prime}(t)+\left(\frac{n-1}{t}\right) u^{\prime}+g(t) f(u)-h(t)\right| \leq \varepsilon,
$$

with the initial condition $u\left(t_{0}\right)=u_{0}, u^{\prime}\left(t_{0}\right)=u_{1}$, then there exists some $z_{0}(t)$ of the equation (1.1) such that

$$
\left|u(t)-z_{0}(t)\right| \leq k \varepsilon
$$

The proof of Corollary 2.1 is quite similar to the proof of Theorem 2.1 and will therefore be omitted.

In the following theorem we establish the HUR stability for (1.1) in the interval $0<t_{0} \leq t \leq T \leq \infty$

Theorem 3.2 Suppose that $u(t) \in C^{(2)}\left(\left[t_{0}, \infty\right),(-\infty, \infty)\right)$ satisfies the inequality (2.1) with initial conditions (1.3). Let $\varphi(t):\left[t_{0}, \infty\right) \rightarrow(0, \infty)$ be a continuous function such that

$$
\int_{t_{0}}^{t} s^{1-n} \int_{t_{0}}^{s} r^{n-1} \varphi(r) d r d s \leq C \varphi(t), \quad \forall t \geq t_{0} .
$$

If there exists a number $a_{0}>0$ such that

$$
|g(t)| \leq \frac{a_{0}}{t^{2}} \text {, for sufficiently large } \mathrm{t} .
$$

then the solution of (1.1) is stable in the sense of HUR as $t \rightarrow \infty$.

Proof. Let $\varepsilon>0$ and $u(t)$ be an approximate solution of the initial value problem (1.1),(1.3). We wish to show that there exists a function

satisfying (1.1) and (1.3) such that

$$
u(t) \in C^{(2)}\left(\left[t_{0}, \infty\right),(-\infty, \infty)\right)
$$

Then it follows from the inequality (2.1) that

$$
\left|u(t)-z_{0}(t)\right| \leq k \varphi(t)
$$

$$
-\varphi(t) \leq u^{\prime \prime}(t)+\left(\frac{n-1}{t}\right) u^{\prime}+g(t) f(u)-h(t) \leq \varphi(t),
$$


Multiplying (3.6) by $t^{n-1}$ gives

$$
-t^{n-1} \varphi(t) \leq\left(t^{n-1} u^{\prime}(t)\right)^{\prime}+t^{n-1} g(t) f(u)-t^{n-1} h(t) \leq t^{n-1} \varphi(t),
$$

Integrating (3.7) with respect to $t$, we have

$$
\begin{aligned}
-\int_{t_{0}}^{t} s^{n-1} \varphi(s) d s & \leq t^{n-1} u^{\prime}(t)-t_{0}{ }^{n-1} u_{1}+\int_{t_{0}}^{t} s^{n-1} g(s) f(u(s)) d s-\int_{t_{0}}^{t} s^{n-1} h(s) d s \\
& \leq \int_{t_{0}}^{t} s^{n-1} \varphi(s) d s,
\end{aligned}
$$

Dividing the last inequality by $t^{n-1}$ we have

$$
\begin{array}{r}
-t^{1-n} \int_{t_{0}}^{t} s^{n-1} \varphi(s) d s \leq u^{\prime}(t)-t^{1-n} t_{0}{ }^{n-1} u_{1}+t^{1-n} \int_{t_{0}}^{t} s^{n-1} g(s) f(u(s)) d s \\
-t^{1-n} \int_{t_{0}}^{t} s^{n-1} h(s) d s \leq t^{1-n} \int_{t_{0}}^{t} s^{n-1} \varphi(s) d s,
\end{array}
$$

If we integrate again the last inequality from $t_{0}$ to $t$, we obtain

$$
\begin{aligned}
\mid u(t)-u_{0}-t^{2-n} t_{0}{ }^{n-1} u_{1} & +t_{0} u_{1}+\int_{t_{0}}^{t} s^{1-n} \int_{t_{0}}^{s} r^{n-1} g(r) f(u(r)) d r d s \\
& \quad-\int_{t_{0}}^{t} s^{1-n} \int_{t_{0}}^{s} r^{n-1} h(r) d r d s \mid \leq \int_{t_{0}}^{t} s^{1-n} \int_{t_{0}}^{s} r^{n-1} \varphi(r) d r d s
\end{aligned}
$$

One can easily show that

$$
\begin{aligned}
z_{0}(t)=u_{0}-t_{0} u_{1}+t^{2-n} t_{0}{ }^{n-1} u_{1} & -\int_{t_{0}}^{t} s^{1-n} \int_{t_{0}}^{s} r^{n-1} g(r) f\left(z_{0}(r)\right) d r d s \\
& -\int_{t_{0}}^{t} s^{1-n} \int_{t_{0}}^{s} r^{n-1} h(r) d r d s
\end{aligned}
$$

satisfies the Eq. (1.1) with the initial condition (1.3).

Now, let us estimate the difference

$$
\begin{aligned}
\left|u(t)-z_{0}(t)\right| \leq \mid u(t)-u_{0}-t^{2-n} t_{0}{ }^{n-1} u_{1}+t_{0} u_{1}+\int_{t_{0}}^{t} s^{1-n} \int_{t_{0}}^{s} r^{n-1} g(r) f(u(r)) d r d s \\
\quad-\int_{t_{0}}^{t} s^{1-n} \int_{t_{0}}^{s} r^{n-1} h(r) d r d s|+| \int_{t_{0}}^{t} s^{1-n} \int_{t_{0}}^{s} r^{n-1} g(r) f(u(r)) d r d s \\
\quad-\int_{t_{0}}^{t} s^{1-n} \int_{t_{0}}^{s} r^{n-1} g(r) f\left(z_{0}(r)\right) d r d s \mid \\
\leq C \varphi(t)+\int_{t_{0}}^{t} s^{1-n} \int_{t_{0}}^{s} r^{n-1}|g(r)|\left|f(u(r))-f\left(z_{0}(r)\right)\right| d r d s
\end{aligned}
$$

By applying the mean value theorem to the integral in the last inequality, and from (1.5) we have

$$
\left|u(t)-z_{0}(t)\right| \leq C \varphi(t)+L\left|g\left(t_{*}\right)\right| \int_{t_{0}}^{t} s^{1-n} \int_{t_{0}}^{s} r^{n-1}\left|u(r)-z_{0}(r)\right| d r d s
$$


Hyers-Ulam-Rassias Stability Criteria of Nonlinear Differential Equations of Lane-Emden Typ

Using Gronwall's inequality we get

$$
\begin{aligned}
\left|u(t)-z_{0}(t)\right| & \leq C \varphi(t) \exp \left(L\left|g\left(t_{*}\right)\right| \int_{t_{0}}^{t} s^{1-n} \int_{t_{0}}^{s} r^{n-1} d r d s\right) \\
& \leq C \varphi(t) \exp \left(\frac{L\left|g\left(t_{*}\right)\right|}{n} \int_{t_{0}}^{t} s^{1-n}\left(s^{n}-t_{0}{ }^{n}\right) d s\right)
\end{aligned}
$$

Now, since $|g(t)| \leq \frac{a_{0}}{t^{2}}$, for sufficiently large $t$ it follows from the last inequality that

$$
\begin{aligned}
\left|u(t)-z_{0}(t)\right| & \leq C \varphi(t) \exp \left(\frac{L}{n t^{2}} \int_{t_{0}}^{t} s^{1-n} s^{n} d s\right)=C \varphi(t) \exp \left(\frac{L}{n t^{2}} \int_{t_{0}}^{t} s d s\right) \\
& \leq C \varphi(t) \exp \left(\frac{L\left(t^{2}-t_{0}^{2}\right)}{2 n t^{2}}\right) \leq C \varphi(t) \exp \left(\frac{L}{2 n}\right) \equiv k \varphi(t)
\end{aligned}
$$

which means that (2.2) holds true for all $t>0$.

Remark 3.1 If we let in Theorems 2.1 and $2.2 h(t) \equiv 0$ then we get the HUR stability for the equation (1.2).

Theorem 3.3 Suppose that $u(t) \in C^{(2)}\left(\left[t_{0}, T\right],(-\infty, \infty)\right)$ satisfies the inequality (2.3) with initial conditions (1.3). Let $\varphi(t):\left[t_{0}, \infty\right) \rightarrow(0, \infty)$ be a continuous function such that

$$
\int_{t_{0}}^{t} s^{-2} \int_{t_{0}}^{s} r^{2} \varphi(r) d r d s \leq C \varphi(t), \quad \forall t \geq t_{0} .
$$

If (1.5) holds, then the solution of (1.4) is stable in the sense of HUR.

Proof. Let $\varepsilon>0$ and $u(t)$ be an approximate solution of the initial value problem (1.4),(1.3). We will show that there exists a function $z_{0}(t) \in C^{(2)}\left(\left[t_{0}, T\right],(-\infty, \infty)\right)$

satisfying (1.4) and (1.3) such that

From the inequality (2.3) we have

$$
\left|u(t)-z_{0}(t)\right| \leq k \varphi(t)
$$

$$
-\varphi(t) \leq u^{\prime \prime}(t)+\left(\frac{2}{t}\right) u^{\prime}+g(t) f(u) \leq \varphi(t)
$$

Multiplying (3.9) by $t^{2}$, we get

$$
-t^{2} \varphi(t) \leq\left(t^{2} u^{\prime}(t)\right)^{\prime}+t^{2} g(t) f(u) \leq t^{2} \varphi(t),
$$

Integrating (3.10) from $t_{0}$ to $t$, we obtain

Or, equivalently

$$
-\int_{t_{0}}^{t} s^{2} \varphi(s) d s \leq t^{2} u^{\prime}(t)-t_{0}^{2} u_{1}+\int_{t_{0}}^{t} s^{2} g(s) f(u(s)) d s \leq \int_{t_{0}}^{t} s^{2} \varphi(s) d s,
$$

$$
\begin{array}{r}
-t^{-2} \int_{t_{0}}^{t} s^{2} \varphi(s) d s \leq u^{\prime}(t)-t^{-2} t_{0}{ }^{n-1} u_{1}+t^{-2} \int_{t_{0}}^{t} s^{2} g(s) f(u(s)) d s \\
\leq t^{-2} \int_{t_{0}}^{t} s^{2} \varphi(s) d s,
\end{array}
$$

Again integrating the last inequality from $t_{0}$ to $t$, we obtain 


$$
\begin{array}{r}
\left|u(t)-u_{0}-t^{-1} t_{0}{ }^{2} u_{1}+t_{0} u_{1}+\int_{t_{0}}^{t} s^{-2} \int_{t_{0}}^{s} r^{2} g(r) f(u(r)) d r d s\right| \\
\leq \int_{t_{0}}^{t} s^{-2} \int_{t_{0}}^{s} r^{2} \varphi(r) d r d s
\end{array}
$$

Clearly we see that

$$
z_{0}(t)=u_{0}-t_{0} u_{1}+t^{-1} t_{0}^{2} u_{1}-\int_{t_{0}}^{t} s^{-2} \int_{t_{0}}^{s} r^{2} g(r) f\left(z_{0}(r)\right) d r d s
$$

satisfies the Eq. (1.4) with the initial condition (1.3).

Now consider the difference

$$
\begin{aligned}
&\left|u(t)-z_{0}(t)\right| \leq\left|u(t)-u_{0}-t^{-1} t_{0}^{2} u_{1}+t_{0} u_{1}+\int_{t_{0}}^{t} s^{-2} \int_{t_{0}}^{s} r^{2} g(r) f(u(r)) d r d s\right| \\
&+\left|\int_{t_{0}}^{t} s^{-2} \int_{t_{0}}^{s} r^{2} g(r) f(u(r)) d r d s-\int_{t_{0}}^{t} s^{-2} \int_{t_{0}}^{s} r^{2} g(r) f\left(z_{0}(r)\right) d r d s\right| \\
& \leq C \varphi(t)+\int_{t_{0}}^{t} s^{-2} \int_{t_{0}}^{s} r^{2}|g(r)|\left|f(u(r))-f\left(z_{0}(r)\right)\right| d r d s \\
& \leq C \varphi(t)+L M \int_{t_{0}}^{t} s^{-2} \int_{t_{0}}^{s} r^{2}\left|u(r)-z_{0}(r)\right| d r d s,
\end{aligned}
$$

where $M=\max _{t_{0 \leq t \leq T}}|g(t)|$.

Applying Gronwall's inequality to above inequality we obtain that

$$
\begin{aligned}
\left|u(t)-z_{0}(t)\right| & \leq C \varphi(t) \exp \left(L M \int_{t_{0}}^{t} s^{-2} \int_{t_{0}}^{s} r^{2} d r d s\right) \\
& \leq C \varphi(t) \exp \left(\frac{L M}{3} \int_{t_{0}}^{t} s^{-2}\left(s^{3}-t_{0}^{3}\right) d s\right) \\
& \leq C \varphi(t) \exp \left(\frac{L M}{3} \int_{t_{0}}^{t} s^{-2} s^{3} d s\right)=C \varphi(t) \exp \left(\frac{L M}{3} \int_{t_{0}}^{t} s d s\right) \\
& \leq C \varphi(t) \exp \left(\frac{L M\left(t^{2}-t_{0}^{2}\right)}{6}\right) \leq C \varphi(t) \exp \left(\frac{L M T^{2}}{6}\right) \equiv k \varphi(t)
\end{aligned}
$$

Thus

$$
\left|u(t)-z_{0}(t)\right| \leq k \varphi(t)
$$

which completes the proof.

Corollary 3.2 Replacing $\varphi(t)$ by $\varepsilon$ in the inequality (3.9) we can get Hyers-Ulam stability for Eq. (1.4) in the interval $0<t_{0} \leq t \leq T$, i.e. if $u(t) \in C^{(2)}\left[t_{0}, T\right]$ satisfies (1.5) and

$$
\left|u^{\prime \prime}(t)+\left(\frac{2}{t}\right) u^{\prime}+g(t) f(u)\right| \leq \varepsilon
$$


Hyers-Ulam-Rassias Stability Criteria of Nonlinear Differential Equations of Lane-Emden Typ

with the initial condition $u\left(t_{0}\right)=u_{0}, u^{\prime}\left(t_{0}\right)=u_{1}$, then there exists some $z_{0}(t)$ of the equation (1.4) such that

$$
\left|u(t)-z_{0}(t)\right| \leq k \varepsilon \text {. }
$$

Since the proof of Corollary 3.2 is very similar to the proof of Theorem 3.3 then it can be omitted.

In the following theorem we establish the HUR stability for (1.4),(1.3) in the interval $0 \leq t_{0}<t<T \leq \infty$

Theorem 3.4 Suppose that $u(t) \in C^{(2)}\left(\left[t_{0}, \infty\right),(-\infty, \infty)\right)$ satisfies the inequality (2.3) with initial conditions (1.3). Let $\varphi(t):\left[t_{0}, \infty\right) \rightarrow(0, \infty)$ be a continuous function such that

$$
\int_{t_{0}}^{t} s^{-2} \int_{t_{0}}^{s} r^{2} \varphi(r) d r d s \leq C \varphi(t), \quad \forall t \geq t_{0} .
$$

If there exists a number $a_{0}>0$ such that

$$
|g(t)| \leq \frac{a_{0}}{t^{2}}, \text { for sufficiently large } \mathrm{t} .
$$

then the solution of (1.4) is stable in the sense of HUR as $t \rightarrow \infty$.

Proof. Let $\varepsilon>0$ and $u(t)$ be an approximate solution of the initial value problem (1.4),(1.3). We will show that there exists a function $z_{0}(t) \in C^{(2)}\left(\left[t_{0}, \infty\right),(-\infty, \infty)\right)$ satisfying (1.4) and (1.3) such that

$$
\left|u(t)-z_{0}(t)\right| \leq k \varphi(t)
$$

From (2.3) the inequality it follows

$$
-\varphi(t) \leq u^{\prime \prime}(t)+\left(\frac{2}{t}\right) u^{\prime}+g(t) f(u) \leq \varphi(t)
$$

The inequality (3.13) can be written as

$$
-t^{2} \varphi(t) \leq\left(t^{2} u^{\prime}(t)\right)^{\prime}+t^{2} g(t) f(u) \leq t^{2} \varphi(t),
$$

Integrating (3.14) successively twice from $t_{0}$ to $t$, one has

$$
\begin{array}{r}
-t^{-2} \int_{t_{0}}^{t} s^{2} \varphi(s) d s \leq u^{\prime}(t)-t^{-2} t_{0}{ }^{2} u_{1}+t^{-2} \int_{t_{0}}^{t} s^{2} g(s) f(u(s)) d s \\
\leq t^{-2} \int_{t_{0}}^{t} s^{2} \varphi(s) d s,
\end{array}
$$

and

$$
\begin{array}{r}
\left|u(t)-u_{0}-t^{-1} t_{0}{ }^{2} u_{1}+t_{0} u_{1}+\int_{t_{0}}^{t} s^{-2} \int_{t_{0}}^{s} r^{2} g(r) f(u(r)) d r d s\right| \\
\leq \int_{t_{0}}^{t} s^{-2} \int_{t_{0}}^{s} r^{2} \varphi(r) d r d s
\end{array}
$$

It can be easily shown that

$$
z_{0}(t)=u_{0}-t_{0} u_{1}+t^{-1} t_{0}^{2} u_{1}-\int_{t_{0}}^{t} s^{-2} \int_{t_{0}}^{s} r^{2} g(r) f\left(z_{0}(r)\right) d r d s
$$

satisfies the Eq. (1.4) with the initial condition (1.3).

Now consider the difference 


$$
\begin{aligned}
\left|u(t)-z_{0}(t)\right| \leq & \left|u(t)-u_{0}-t^{-1} t_{0}^{2} u_{1}+t_{0} u_{1}+\int_{t_{0}}^{t} s^{-2} \int_{t_{0}}^{s} r^{2} g(r) f(u(r)) d r d s\right| \\
& +\left|\int_{t_{0}}^{t} s^{-2} \int_{t_{0}}^{s} r^{2} g(r) f(u(r)) d r d s-\int_{t_{0}}^{t} s^{-2} \int_{t_{0}}^{s} r^{2} g(r) f\left(z_{0}(r)\right) d r d s\right| \\
& \leq C \varphi(t)+\int_{t_{0}}^{t} s^{-2} \int_{t_{0}}^{s} r^{2}|g(r)|\left|f(u(r))-f\left(z_{0}(r)\right)\right| d r d s
\end{aligned}
$$

By applying the mean value theorem to the integral in the last inequality and from (1.5), we have

$$
\left|u(t)-z_{0}(t)\right| \leq C \varphi(t)+L\left|g\left(t_{*}\right)\right| \int_{t_{0}}^{t} s^{-2} \int_{t_{0}}^{s} r^{2}\left|u(r)-z_{0}(r)\right| d r d s
$$

Using Gronwall's inequality yields

$$
\begin{aligned}
\left|u(t)-z_{0}(t)\right| & \leq C \varphi(t) \exp \left(L\left|g\left(t_{*}\right)\right| \int_{t_{0}}^{t} s^{-2} \int_{t_{0}}^{s} r^{2} d r d s\right) \\
& \leq C \varphi(t) \exp \left(\frac{L\left|g\left(t_{*}\right)\right|}{n} \int_{t_{0}}^{t} s^{-2}\left(s^{2}-t_{0}{ }^{2}\right) d s\right)
\end{aligned}
$$

In view of condition (3.12) it follows that for sufficiently large t, say $t \geq t_{0}$

$$
\begin{aligned}
\left|u(t)-z_{0}(t)\right| & \leq C \varphi(t) \exp \left(\frac{L}{3 t^{2}} \int_{t_{0}}^{t} s^{-2} s^{3} d s\right)=C \varphi(t) \exp \left(\frac{L}{3 t^{2}} \int_{t_{0}}^{t} s d s\right) \\
& \leq C \varphi(t) \exp \left(\frac{L\left(t^{2}-t_{0}^{2}\right)}{6 t^{2}}\right) \leq C \varphi(t) \exp \left(\frac{L}{6}\right) \equiv k \varphi(t)
\end{aligned}
$$

which establishes the HUR stability for arbitrarily large $t$.

\section{Special Cases of Equation (1.4)}

In this section we consider HUR stability for the most typical of Lane-Emden equation

$$
u^{\prime \prime}(t)+\left(\frac{2}{t}\right) u^{\prime}+u^{m}=0, m \geq 1
$$

and the white dwarf equation

$$
u^{\prime \prime}(t)+\left(\frac{2}{t}\right) u^{\prime}+[u(u+1)]^{3 / 2}=0
$$

with the initial conditions

$$
u\left(t_{0}\right)=u_{0}, u^{\prime}\left(t_{0}\right)=u_{1}
$$

Theorem 4.1 Suppose that $u(t) \in C^{(2)}\left(\left[t_{0}, T\right],(-\infty, \infty)\right)$ satisfies the inequality (3.1) with initial conditions (4.3). If $\varphi(t):\left[t_{0}, \infty\right) \rightarrow(0, \infty)$ is a continuous function such that

$$
\int_{t_{0}}^{t} s^{-2} \int_{t_{0}}^{s} r^{2} \varphi(r) d r d s \leq C \varphi(t), \quad \forall t \geq t_{0} .
$$

then the solution of the equation (4.1) is stable in the sense of HUR.

Proof. Let $\varepsilon>0$ and $u(t)$ be an approximate solution of the initial value problem (4.1),(4.3). We will show that there exists a function $z_{0}(t) \in C^{(2)}\left(\left[t_{0}, T\right],(-\infty, \infty)\right)$ 
Hyers-Ulam-Rassias Stability Criteria of Nonlinear Differential Equations of Lane-Emden Typ

satisfying (4.1) and (4.3) such that

$$
\left|u(t)-z_{0}(t)\right| \leq k \varphi(t)
$$

Since $u(t)$ is an approximate solution of Eq. (4.1), then we get the inequality

$$
-\varphi(t) \leq u^{\prime \prime}(t)+\left(\frac{2}{t}\right) u^{\prime}+u^{m} \leq \varphi(t)
$$

Multiplying (4.4) by $t^{2}$, we get

$$
-t^{2} \varphi(t) \leq\left(t^{2} u^{\prime}(t)\right)^{\prime}+t^{2} u^{m} \leq t^{2} \varphi(t)
$$

Integrating (4.5) from $t_{0}$ to $t$, we have

Or, equivalently

$$
-\int_{t_{0}}^{t} s^{2} \varphi(s) d s \leq t^{2} u^{\prime}(t)-t_{0}^{2} u_{1}+\int_{t_{0}}^{t} s^{2} u^{m}(s) d s \leq \int_{t_{0}}^{t} s^{2} \varphi(s) d s,
$$

$$
\begin{aligned}
-t^{-2} \int_{t_{0}}^{t} s^{2} \varphi(s) d s \leq u^{\prime}(t)-t^{-2} t_{0}{ }^{n-1} u_{1}+t^{-2} \int_{t_{0}}^{t} s^{2} u^{m}(s) d s & \\
& \leq t^{-2} \int_{t_{0}}^{t} s^{2} \varphi(s) d s,
\end{aligned}
$$

Again integrating the last inequality from $t_{0}$ to $t$, we obtain

$$
\begin{array}{r}
\left|u(t)-u_{0}-t^{-1} t_{0}{ }^{2} u_{1}+t_{0} u_{1}+\int_{t_{0}}^{t} s^{-2} \int_{t_{0}}^{s} r^{2} u^{m}(r) d r d s\right| \\
\leq \int_{t_{0}}^{t} s^{-2} \int_{t_{0}}^{s} r^{2} \varphi(r) d r d s
\end{array}
$$

It is clear to see that

$$
z_{0}(t)=u_{0}-t_{0} u_{1}+t^{-1} t_{0}{ }^{2} u_{1}-\int_{t_{0}}^{t} s^{-2} \int_{t_{0}}^{s} r^{2} z_{0}{ }^{m}(r) d r d s
$$

satisfies the Eq. (4.1) with the initial condition (4.3).

Let us consider the difference

$$
\begin{aligned}
\left|u(t)-z_{0}(t)\right| \leq & \left|u(t)-u_{0}-t^{-1} t_{0}^{2} u_{1}+t_{0} u_{1}+\int_{t_{0}}^{t} s^{-2} \int_{t_{0}}^{s} r^{2} u^{m}(r) d r d s\right| \\
& +\left|\int_{t_{0}}^{t} s^{-2} \int_{t_{0}}^{s} r^{2} u^{m}(r) d r d s-\int_{t_{0}}^{t} s^{-2} \int_{t_{0}}^{s} r^{2} z_{0}{ }^{m}(r) d r d s\right| \\
& \leq C \varphi(t)+\int_{t_{0}}^{t} s^{-2} \int_{t_{0}}^{s} r^{2}|g(r)|\left|u^{m}(r)-z_{0}{ }^{m}(r)\right| d r d s
\end{aligned}
$$

Now, since $u(t) \in C^{(2)}\left(\left[t_{0}, T\right],(-\infty, \infty)\right)$, then $\left|\frac{d\left(u^{m}\right)}{d u}\right|$ is bounded and hence the function $u^{m}$ satisfies Lipschitz condition in $u$ for all $t \in\left[t_{0}, T\right]$ and $m \geq 1$.

\section{Consequently}




$$
\left|u(t)-z_{0}(t)\right| \leq C \varphi(t)+L \int_{t_{0}}^{t} s^{-2} \int_{t_{0}}^{s} r^{2}\left|u(r)-z_{0}(r)\right| d r d s,
$$

From the Gronwall's inequality, we obtain

$$
\begin{aligned}
\left|u(t)-z_{0}(t)\right| & \leq C \varphi(t) \exp \left(L \int_{t_{0}}^{t} s^{-2} \int_{t_{0}}^{s} r^{2} d r d s\right) \\
& \leq C \varphi(t) \exp \left(\frac{L}{3} \int_{t_{0}}^{t} s^{-2}\left(s^{3}-t_{0}{ }^{3}\right) d s\right) \\
& \leq C \varphi(t) \exp \left(\frac{L}{3} \int_{t_{0}}^{t} s^{-2} s^{3} d s\right)=C \varphi(t) \exp \left(\frac{L}{3} \int_{t_{0}}^{t} s d s\right) \\
& \leq C \varphi(t) \exp \left(\frac{L\left(t^{2}-t_{0}{ }^{2}\right)}{6}\right) \leq C \varphi(t) \exp \left(\frac{L T^{2}}{6}\right)
\end{aligned}
$$

Thus

$$
\left|u(t)-z_{0}(t)\right| \leq k \varphi(t)
$$

i.e. equation (4.1) is HUR stable.

Corollary 4.1 Replacing $\varphi(t)$ by $\varepsilon$ in the inequality (4.5) we get Hyers-Ulam stability for Eq. (4.1) in the interval $0<t_{0} \leq t \leq T$.

Theorem 4.2 Suppose that $u(t) \in C^{(2)}\left[\left[t_{0}, T\right],(-\infty, \infty)\right]$ satisfies the inequality (4.2) with initial conditions (4.3). If $\varphi(t):\left[t_{0}, \infty\right) \rightarrow(0, \infty)$ is a continuous function such that

$$
\int_{t_{0}}^{t} s^{-2} \int_{t_{0}}^{s} r^{2} \varphi(r) d r d s \leq C \varphi(t), \quad \forall t \geq t_{0}
$$

then the solution of the equation (4.2) is stable in the sense of HUR.

It should be noted here that the proof of Theorem 4.2 is very similar to the proof of Theorem 4.1 and it will therefore be omitted.

\section{Conclusion}

In this work, the problem of the HUR Stability of solutions of nonlinear differential equations of Lane-Emden type has been investigated and solved using the direct method. To illustrate the results we provided the most two typical examples satisfying the assumptions of the proved theorems.

\section{References}

[1] Alsina C., Ger R. (1998). 'On some inequalities and stability results related to the exponential function', Journal of Inequalities and Application 2, pp 373-380.

[2] Gavruta P. (1994). ' A generalization of the Hyers-Ulam-Rassias stability of approximately additive mappings', J. Math. Anal. and Appl., Vol. 184, No. 3, pp. 431--436.

[3] Gavruta P., Jung S. and Li Y.(2011) 'Hyers-Ulam Stability for Second-Order Linear differential Equations with Boundary Conditions', EJDE. 80:1-7, http://ejde.math.txstate.edu/ Volumes/2011/ 80/gavruta.pdf.

[4] Gordji M. E, Cho Y. J., Ghaemi M. B. and Alizadeh B., (2011) 'Stability of the exact second order partial differential equations', J. Inequal. Appl. Article ID: 306275.

[5] Hyers D. H. (1941) 'On the stability of the linear functional equation', Proceedings of the National Academy of Sciences of the United States of America, vol. 27, pp. 222-224. 
Hyers-Ulam-Rassias Stability Criteria of Nonlinear Differential Equations of Lane-Emden Typ

[6] Jun K .-W., Lee Y. -H., (2004). 'A generalization of the Hyers-Ulam-Rassias stability of the Pexiderized quadratic equations', Journal of Mathematical Analysis and Applications , vol. 297, no.1, pp. 70-86.

[7] Jung S. M. (1996) 'On the Hyers-Ulam-Rassias stability of approximately additive mappings', J. Math. Anal. Appl.204, pp. 221-226.

[8] Jung S. M. (2001). ' Hyers-Ulam-Rassias Stability of Functional Equations in Mathematical Analysis', Hadronic Press, Palm Harbor, USA.

[9] Jung S. M. (2006). ' Hyers-Ulam stability of a system of first order linear differential equations with constant coefficients, Journal of Mathematical Analysis and Applications', vol. 320, no. 2, pp. 59--561.

[10] Li Y. (2010). 'Hyers-Ulam Stability of Linear Differential Equations', Thai J. Math., Vol. 8 No 2, pp 215--219.

[11] Li Y. and Shen Y. (2009). ' Hyers-Ulam Stability of Nonhomogeneous Linear Differential Equations of Second Order', Internat. J. Math. Math. Sci., Vol. 2009, pp. 7, Article ID 576852.

[12] Lungu N. and Craciun C. (2012). ' Ulam-Hyers-Rassias Stability of a Hyperbolic Partial Differential Equation', ISRN Math. Anal., Volume 2012, Article ID 609754, 10 pages, doi:10.5402/2012/609754

[13] Miura T., Takahasi S.-E and Choda. H. (2001) . ' On the Hyers-Ulam stability of real continuous function valued differentiable map', Tokyo J. Math, 4, pp. 467- 476.

[14] Miura T., Miyajima S., Takahasi S.-E. (2007) . ' A characterization of Hyers- Ulam stability of first order linear differential operators', J. Math. Anal. Appl.286, pp.136-146.

[15] Obloza M. (1993). ' Hyers stability of the linear differential equation' , Rocznik Nauk.Dydakt. Prace Mat., No. 13, 259-270.

[16] Obloza M. (1997). ' Connections between Hyers and Lyapunov stability of the ordinary differential equations', Rocznik Nauk.-Dydakt. Prace Mat., No. 14, 141-146.

[17] Okunuga, S. A.; Ehigie, J. O.; Sofoluwe, A. B. (2012). ' Treatment of Lane- Emden Type Equations via Second Derivative Backward Differentiation Formula using Boundary Value Technique', // Proceedings of the World Congress on Engineering 2012 Volume I, p224.

[18] Park C. G. (2002). ' On the stability of the linear mapping in Banach modules', J. Math. Anal. Appl.275, pp. 711-720.

[19] Park C. G. (2005) . ' Homomorphisms between Poisson JC*-algebras', Bull. Braz. Math. Soc., vol. 36, no. 1 , pp. 79--97.

[20] Park C. G., Cho Y.-S. and Han M. (2007). ' Functional inequalities associated with Jordan-von Neumann type additive functional equations', J. Inequal. Appl.,vol. 2007, pp. 13 , Article ID 41820.

[21] Popa D and Rus I. (2011). ' On the Hyers-Ulam stability of the linear differential equation', Journal of Mathematical Analysis and Applications, 381(2): 530 - 537.

[22] Popa D and Rus I.( 2012). ' Hyers-Ulam stability of the linear differential operator with nonconstant coefficients', Applied Mathematics and Computation; 219(4) : 1562-1568.

[23] Rassias T. M. (1978) . ' On the stability of the linear mapping in Banach spaces',Proc. Amer. Math. Soc., vol. 72, no. 2, pp. 297-300.

[24] Rus I. (2009). ' Remarks on Ulam stability of the operatorial equations', Fixed Point Theory, vol. 10, no. 2, pp. 305-320.

[25] Rus I . (2009). Ulam stability of ordinary differential equations,Studia Universitatis BabesBolyai: Mathematica, vol. 5 , no., pp. 125--133. 
[26] Takahasi E., Miura T., and Miyajima S. (2002). 'On the Hyers-Ulam stability of the Banach space-valued differential equation $y^{\prime}=\lambda y^{\prime}$, Bull. Korean Math. Soc., Vol. 39, No. 2, pp 309--315. [27] Ulam S.M. (1964).' Problems in Modern Mathematics', John Wiley \& Sons, New York, USA, Science edition.

[28] Wang G., Zhou M. and Sun L. (2008). 'Hyers-Ulam stability of linear differential equations of first order', Appl. Math. Lett., 21, pp 1024-1028. 